\title{
PDE4 and PDE5 regulate cyclic nucleotides relaxing effects in human umbilical arteries
}

\author{
António José Santos-Silva ${ }^{\mathrm{a}}$, Elisa Cairrão ${ }^{\mathrm{a}, \mathrm{b}}$, Manuel Morgado ${ }^{\mathrm{a}, \mathrm{b}}$, \\ Ezequiel Álvarez ${ }^{a}$, Ignacio Verde ${ }^{\mathrm{a}, *}$ \\ ${ }^{a}$ CICS - Centro de Investigação em Ciências da Saúde, Universidade da Beira Interior, Av. Infante D. Henrique, 6200-506 Covilhã, Portugal \\ ${ }^{\mathrm{b}}$ Centro Hospitalar da Cova da Beira E.P.E. Quinta do Alvito, 6200-251 Covilhã, Portugal
}

Received 27 July 2007; received in revised form 21 November 2007; accepted 16 December 2007

Available online 28 December 2007

\begin{abstract}
Cyclic nucleotides (cAMP and cGMP) are the main second messengers linked to vasodilatation. They are synthesized by cyclases and degraded by different types of phosphodiesterases (PDE). The effect of PDE inhibition and cyclases stimulation on 5-hydroxytryptamine (5-HT; $1 \mu \mathrm{M})$ and histamine $(10 \mu \mathrm{M})$ contracted arteries was analysed. Stimulation of guanylate cyclase or adenylate cyclase relaxed the histamine- and 5-HT-induced contractions indicating that intracellular increase of cyclic nucleotides leads to vasodilatation of the human umbilical artery. We investigated the role of different PDE families in the regulation of this effect. The presence of the different PDE types in human umbilical artery smooth muscle was analysed by RT-PCR and the expression of PDE1B, PDE3A, PDE3B, PDE4C, PDE4D and PDE5A was detected. The unspecific PDE inhibitor 3-isobutyl-1-methylxanthine (IBMX; $50 \mu \mathrm{M}$ ) relaxed histamine-contracted human umbilical artery on 47.4 $\pm 7.2 \%$. This effect seems to be due to PDE4 and PDE5 inhibition because among the selective PDE inhibitors used only the PDE4 inhibitor (rolipram; $1 \mu \mathrm{M}$ ) and the PDE5 inhibitors (dipyridamole and T0156; $3 \mu \mathrm{M}$ and $1 \mu \mathrm{M}$ respectively) induced significant relaxation $(39.0 \pm 8.7,30.4 \pm 6.0$ and $36.3 \pm$ 2.8 respectively). IBMX, dipyridamole and T0156 produced similar relaxation on 5-HT-induced contraction. After forskolin, the addition of IBMX or rolipram increased the effect of the adenylate cyclase stimulator and almost completely relaxed the human umbilical artery contracted by histamine (92.5 \pm 4.9 and $90.9 \pm 4.7$ respectively), suggesting a main role of PDE4. The data obtained with 5-HT contracted arteries confirmed this, because only rolipram and IBMX significantly increased the forskolin vasodilator effect. The administration of dipyridamole and T0156 after sodium nitroprusside (SNP) induced a significant increase of the SNP relaxant effect on histamine-contracted arteries, but PDE1 and PDE3 inhibition did not increase the effect of the guanylate cyclase stimulator. Similar effects were obtained in 5-HT contracted arteries, the SNP induced relaxation was increased by the PDE5 inhibition, but not by PDE1 or PDE3 inhibition. In summary, our results demonstrate that: 1) the increase of cAMP and/or cGMP levels induces relaxation of the human umbilical vascular smooth muscle; 2) four families of PDE are expressed in this smooth muscle: PDE1, PDE3, PDE4 and PDE5; 3) between these families, PDE4 and PDE5 are the key enzymes involved in the regulation of the relaxation associated to cAMP and cGMP, respectively.
\end{abstract}

(C) 2007 Elsevier B.V. All rights reserved.

Keywords: Cyclic nucleotide; Phosphodiesterase; Human umbilical artery; Smooth muscle

\section{Introduction}

Human umbilical artery is involved in fetoplacental circulation. The mechanisms that regulate the contractile state of this artery are very important for optimum gas and nutrient exchange between the foetus and the placenta. Since the umbilical blood

\footnotetext{
* Corresponding author. Tel.: +351 275 329049; fax: +351 275329099 .

E-mail address: iverde@fcsaude.ubi.pt (I. Verde).
}

vessels are not innervated, the control of umbilical blood flow depends entirely on vasoactive substances either released locally or existing in the circulation (Leung et al., 2006). The understanding of the intracellular mechanisms modulating human umbilical artery contractility may offer therapeutic value for the treatment of some pathologies as pre-eclampsia. Cyclic nucleotides (cAMP and cGMP) are the main second messengers linked to vasodilatation. The intracellular levels of these second messengers are regulated by adenylate cyclase and guanylate cyclase, 
responsible of the cyclic nucleotides synthesis, and phosphodiesterases (PDEs), involved in their hydrolysis (Lugnier, 2006; Rybalkin et al., 2003; Tilley and Maurice, 2002). Because the PDE isoenzymes are differentially expressed and regulated, they were considered good targets for therapeutic intervention for cardiovascular diseases (Matsumoto et al., 2003; Rybalkin et al., 2003), but additional knowledge is necessary to achieve this point. Concerning the fetoplacental circulation, cyclic nucleotides increase was appointed as beneficial because it dilates the arteries, enhances placental oxygen uptake alleviating hypoxemic fetal stress and ameliorates the fetal nutrition resumes (Downing et al., 2004). In this sense, nitrovasodilators were already suggested for the treatment of hypertension in pregnancy (Amit et al., 1998).

To date, at least 11 different families of PDE isozymes have been identified, which are further differentiated into 24 subfamilies with various splice variants (Gupta et al., 2005; Lugnier, 2006; Matsumoto et al., 2003). The PDE isozymes described as being expressed in distinct types of vascular smooth muscle are PDE1, PDE3, PDE4 and PDE5 (Maurice et al., 2003; Noguera et al., 2001; Rybalkin et al., 2003). PDE2 and PDE7 transcripts were only detected in porcine aorta smooth muscle and in human pulmonary artery respectively (Matsumoto et al., 2003).

These PDEs families differ in their regulation and substrate specificity (cAMP or cGMP) (Lugnier, 2006; Matsumoto et al., 2003). PDE1 is a $\mathrm{Ca}^{2+}$-calmodulin-activated PDE which hydrolyses cAMP and cGMP (Beavo, 1995; Lugnier, 2006). Three different PDE1 gene products have been cloned (PDE1A, PDE1B and PDE1C). PDE1A and PDE1B hydrolyse cGMP more efficiently than cAMP (Beavo, 1995) and have been identified in several vascular smooth muscles (Matsumoto et al., 2003). PDE1C hydrolyses cAMP and cGMP with equal efficiency but is only expressed in cultured smooth muscle cells and its function is relevant for cell proliferation (Maurice et al., 2003; Noguera et al., 2001; Rybalkin et al., 2003). PDE2 is encoded by a unique gene and is a cGMP-stimulated PDE which hydrolyses cAMP and cGMP (Gupta et al., 2005; Lugnier, 2006; Matsumoto et al., 2003). PDE3 is a cGMP-inhibited PDE which hydrolyses cAMP with a rate 10-fold greater than for cGMP hydrolysis, but this enzyme has a greater affinity for cGMP (Beavo, 1995; Gupta et al., 2005; Lugnier, 2006). At present, two genes of this family were described: PDE3A and PDE3B and products of both genes were identified in the several vascular smooth muscle cells (Gupta et al., 2005; Lugnier, 2006). PDE4 hydrolyses selectively cAMP with high affinity and is specifically inhibited by rolipram (Gupta et al., 2005; Lugnier, 2006; Matsumoto et al., 2003). PDE4 is currently the largest family of PDEs and products of four different genes are known (PDE4A, PDE4B, PDE4C and PDE4D) (Gupta et al., 2005; Lugnier, 2006). PDE5 specifically hydrolyses cGMP with high affinity (Matsumoto et al., 2003). High levels of PDE5 were found in several smooth muscles, including vascular smooth muscle (Gupta et al., 2005). To date, only one gene has been reported for this family (PDE5A) (Gupta et al., 2005). PDE7 specifically hydrolyses cAMP with high affinity (Matsumoto et al., 2003). In humans and mice, two genes (PDE7A and PDE7B) have been identified (Matsumoto et al., 2003).

The increase in the intracellular levels of cyclic nucleotides by cyclases stimulation or by PDEs inhibition represents a useful strategy to study their biological effects. Relaxation of vascular smooth muscle induced by PDE inhibitors was described by several authors. Some authors indicated that PDE1 and PDE3 play an important role modulating the contractility of rat aorta (Noguera et al., 2001) and other observed that PDE5 inhibition induces direct relaxation of this artery (Mochida et al., 2002a). Lillestll et al. (1998) demonstrated that PDE3 was the main enzyme regulating the cGMP-relaxant effect in porcine coronary artery (Lillestll et al., 1998). Willette et al. suggested that PDE4 is the predominant family implicated in the cAMPdependent regulation of the canine basilar artery contractility (Willette et al., 1997). Waldkirch et al. observed that cAMPdependent relaxation is mediated by PDE3 and PDE4 and PDE5 is the main family modulating the vasodilatation associated to cGMP in human cavernous arteries (Waldkirch et al., 2005). The involvement of PDE3 and PDE5 in the modulation of cGMP-dependent relaxation was also observed in human pulmonary artery (Rabe et al., 1994). Due to the cGMP effects in fetoplacental circulation, some authors proposed the use of nitrovasodilators or PDE5 inhibitors for pregnant women with vascular complications (Amit et al., 1998; Downing et al., 2004; Pinheiro da Costa et al., 2006). Furthermore, nitric oxide (NO) and sodium nitroprusside (NO donor) were shown to induce relaxation of the human umbilical artery (Lovren and Triggle, 2000). This biological action of NO can be attributed to the stimulation of soluble guanylate cyclase and the subsequent production of cGMP (Lovren and Triggle, 2000).

The purpose of the present study was to investigate the involvement of the cyclic nucleotides (cAMP and cGMP) and PDEs in the mechanisms of human umbilical artery relaxation. We investigated the expression of different PDE isoforms in smooth muscle layers of this artery. The effects of several PDE inhibitors, either alone and in the presence of adenylate cyclase or guanylate cyclase stimulators, on the contractions induced by histamine and 5-HT in denuded human umbilical artery were also studied.

\section{Methods}

\subsection{Tissue preparation}

Umbilical cord pieces of 3-7 cm were obtained from normal term pregnancies with the consent of the donor mothers. All procedures carried out with these samples were approved by the Ethics Committee of "Centro Hospitalar da Cova da Beira EPE". The umbilical cord samples were collected in sterile physiological saline solution (composition, $\mathrm{mM}: \mathrm{NaCl} 110 ; \mathrm{CaCl}_{2}$ $0.15 ; \mathrm{KCl} 5 ; \mathrm{MgCl}_{2}$ 2; HEPES 10; $\mathrm{NaHCO}_{3} 10 ; \mathrm{KH}_{2} \mathrm{PO}_{4}$ 0.5; $\mathrm{NaH}_{2} \mathrm{PO}_{4}$ 0.5; Glucose 10; EDTA 0.49). In order to avoid contamination and tissue degradation, penicillin $(5 \mathrm{U} / \mathrm{ml})$, streptomycin $(5 \mu \mathrm{g} / \mathrm{ml})$, amphotericin B $(12.5 \mathrm{ng} / \mathrm{ml})$ and antiproteases (leupeptine, $0.45 \mathrm{mg} / \mathrm{l}$; benzamidine, $26 \mathrm{mg} / \mathrm{l}$; and trypsin inhibitor, $10 \mathrm{mg} / \mathrm{l}$ ) were added to the physiological saline solution. Umbilical artery rings of $3-5 \mathrm{~mm}$ were isolated from the surrounding connective tissue. Vascular endothelium was mechanically removed by gentle rubbing with a cotton bud introduced through the arterial lumen. Some rings were chopped to 
rectangle pieces using scissors and smooth muscle layers of the tunica media of the artery were extracted using surgical forceps and scalpel. The reverse transcriptase PCR experiments were performed using RNA extracted from these samples.

\subsection{PDEs expression analysis by reverse transcriptase PCR}

Reverse transcriptase protein chain reaction (RT-PCR) by using primers against the different PDE isoforms was used to analyse their expression. The human housekeeping gene glyceraldehyde 3-phosphate dehydrogenase (GAPDH) was used to assess relative quantities of DNA from each sample.

Total RNA was prepared from smooth muscle layers of human umbilical artery using the Tri reagent ${ }^{\mathrm{TM}}$ RNA purification system (Sigma, Saint Louis, USA). The cDNA was prepared from mRNA with random primers $(3 \mu \mathrm{g} / \mu \mathrm{l})$ using Superscipt ${ }^{\mathrm{TM}}$ II RNAase H-reverse transcriptase and the conditions recommended by the manufacturer $(10 \mathrm{U} / \mu \mathrm{l})$. Five ng of cDNA was amplified in $25 \mu 1$ reaction mixture containing $200 \mu \mathrm{M}$ dNTPs, $0.625 \mathrm{U}$ of Taq polymerase and $25 \mathrm{pmol} / \mathrm{ml}$ of primers in the buffer supplied by the manufacturer (Promega, Madison, USA). Oligonucleotide primers designed were as follows:

PDE1A (Genbank accession number U40370): Forward primer 5'-gtccetgattctccacgcagcag-3', reverse primer 5'-tggt ggttgagctgcttgccac- $3^{\prime}$, fragment size $314 \mathrm{bp}$.

PDE1B (Genbank accession number AJ401609): Forward primer $5^{\prime}$-tctgctccaagggagcgtggtc- $3^{\prime}$, reverse primer $5^{\prime}$-tgc cagctaagatctggggtgg- $3^{\prime}$, fragment size $366 \mathrm{bp}$.

PDE2A (Genbank accession number U67733): Forward primer $5^{\prime}$-gtggagaagcataccctggtcg-3', reverse primer 5'-ggag attgtcctccgacaccagc- $3^{\prime}$, fragment size $280 \mathrm{bp}$.

PDE3A (Genbank accession number M91667): Forward primer $5^{\prime}$-tgatcgecttgactagcgcggtc-3'，5'-tccetcggtatacaggg cagg- $3^{\prime}$, fragment size $316 \mathrm{bp}$.

PDE3B (Genbank accession number U38178): Forward primer $5^{\prime}$-tgctgagctgcgtagggctgctg-3', reverse primer 5'actgccatagtaactggctgcag-3', fragment size $332 \mathrm{bp}$

PDE4A (Genbank accession number L20965): Forward primer $5^{\prime}$-tctgtcagcgagatggectcg-3', reverse primer $5^{\prime}$-ggct gttatggtaggccacgtc- $3^{\prime}$, fragment size $550 \mathrm{bp}$.

PDE4B (Genbank accession number L20966): Forward primer $5^{\prime}$-tccaagcgagcaacacggcgatg- $3^{\prime}$, reverse primer $5^{\prime}$-tga gaggtgtgtcagctcccggt- $3^{\prime}$, fragment size $322 \mathrm{bp}$.

PDE4C (Genbank accession number Z46632): Forward primer $5^{\prime}$-tcagttggagacgctgcagacc- $3^{\prime}$, reverse primer $5^{\prime}$-gta ggccactgatccgggacatg-3', fragment size $227 \mathrm{bp}$.

PDE4D (Genbank accession number L20970): Forward primer $5^{\prime}$-accagaaactggecagcgagac- $3^{\prime}$, reverse primer $5^{\prime}$-aga gagatgggtgagctccega- $3^{\prime}$, fragment size $143 \mathrm{bp}$.

PDE5A (Genbank accession number AF043731): Forward primer $5^{\prime}$-gacatgtggcagcgettggtg- $3^{\prime}$, reverse primer $5^{\prime}$-agca aggtcaagcagcacctga- $3^{\prime}$, fragment size $320 \mathrm{bp}$.

PDE7A1 (Genbank accession number L12052): Forward primer $5^{\prime}$-ggcagggcgggcgtattca-3', reverse primer $5^{\prime}$-catgg cetgagtaacatccgc- $3^{\prime}$, fragment size $690 \mathrm{bp}$.
PDE7A2 (Genbank accession number U67932): Forward primer $5^{\prime}$-attgatctggtgtctggccttgg- $3^{\prime}$, reverse primer $5^{\prime}$-catgg cctgagtaacatccgc-3', fragment size $582 \mathrm{bp}$.

GAPDH (Genbank accession number AF261085): Forward primer 5'-tgaaggtcggagtcaacggatttggt- $3^{\prime}$, reverse primer $5^{\prime}$ catgtgggccatgaggtccaccac- $3^{\prime}$, fragment size $983 \mathrm{bp}$.

The PCR procedures were performed as follows: 30 cycles (30 s at $94{ }^{\circ} \mathrm{C}, 30 \mathrm{~s}$ at $55-60{ }^{\circ} \mathrm{C}$ and $30 \mathrm{~s}$ at $72{ }^{\circ} \mathrm{C}$ ) and a final elongation $\left(5 \mathrm{~min}\right.$ at $\left.72^{\circ} \mathrm{C}\right)$. The PCR products were separated by electrophoresis on $2 \%$ agarose gel containing $0.01 \%$ of ethidium bromide and photographed under UV irradiation at $320 \mathrm{~nm}$.

\subsection{Artery tension recording}

The human umbilical artery rings were placed in an organ bath (LE01.004, Letica) containing Krebs-bicarbonate solution (composition in mM: $\mathrm{NaCl} 119, \mathrm{KCl} 5.0, \mathrm{NaHCO}_{3} 25, \mathrm{KH}_{2} \mathrm{PO}_{4}$ $1.2, \mathrm{CaCl}_{2} 0.5, \mathrm{MgSO}_{4} 1.2$, EDTA 0.03 , Glucose 11 ) at $37^{\circ} \mathrm{C}$ and continuously gassed with carbogen. The artery rings were suspended by two parallel stainless steel wires and tension measurement was performed using isometric transducers (TRI201, Panlab, Spain), amplifier (ML118/D Quad Bridge, ADInstruments), interface PowerLab/4SP (ML750, ADInstruments) and computerised system with Chart5 PowerLab software (ADInstruments). During the resting periods, the organ bath solution was changed every $15 \mathrm{~min}$. Initially, the rings were equilibrated for 60 min until a resting tension of $1 \mathrm{~g}$ was achieved. After this, the rings were challenged with 5 -HT $(1 \mu \mathrm{M})$ to test their viability. Rings that induced contraction lower than $1 \mathrm{~g}$ when challenged with 5-HT were excluded from the study. Afterwards, the rings were contracted using histamine $(10 \mu \mathrm{M})$ or 5 -HT $(1 \mu \mathrm{M})$ and the role of PDE isoforms in cyclic nucleotide mediated vasodilatation was evaluated using different PDE inhibitors. 3-isobutyl-1methylxanthine (IBMX, $50 \mu \mathrm{M}$ ) was used as a broad-spectrum PDE inhibitor. A $20 \mu \mathrm{M}$ concentration of 8-methoxymethyl-3isobutyl-1-methylxantine (MIBMX) was employed to selectively inhibit PDE1. To selective inhibit PDE3 cilostamide $1 \mu \mathrm{M}$ was utilised. Rolipram $1 \mu \mathrm{M}$ was used to selective inhibit PDE4. The selective inhibition of PDE5 was obtained using dipyridamole $3 \mu \mathrm{M}$ or T0156 $1 \mu \mathrm{M}$.

The effects of forskolin (adenylate cyclase stimulator) and sodium nitroprusside (SNP; guanylate cyclase stimulator) on 5HT- and histamine-contracted human umbilical arteries were also studied. The effects of these cyclases stimulators were also analysed in presence of several PDE inhibitors to determine the role of these enzymes in the control of the cAMP and cGMP levels.

\subsection{Drugs and chemicals}

All drugs and chemicals were purchased from SigmaAldrich Quimica (Sintra, Portugal), except forskolin, rolipram, T0156 and ciclostamide, which were purchased from Biogen Cientifica (Madrid, Spain). Forskolin, rolipram, IBMX, dipyridamole, MIBMX and ciclostamide were initially dissolved in ethanol. Histamine, 5-HT and SNP were initially dissolved in 
distilled water. Final solutions were obtained by dilution with Krebs solution. The final concentration of ethanol in the organ bath did not exceed $0.1 \%$.

\subsection{Statistical analysis}

Statistical analysis of the data was performed using the SigmaStat Statistical Analysis System, version 1.00 (1992). Results were expressed as mean \pm S.E.M. of $n$ experiments. Comparison among multiple groups was analysed by using a one-way ANOVA followed by Dunnet's post hoc test to determine significant differences among the means. Probability levels lower than 5\% were considered significant $(P<0.05)$.

\section{Results}

\subsection{Expression of PDE in human umbilical artery}

Semi-quantitative RT-PCR analysis using specific primers for several human PDE genes was performed in human umbilical artery smooth muscle layers in order to check for the expression of PDE1 (A and B), PDE2A, PDE3 (A and B), PDE4 (A, B, C and D) PDE5A and PDE7 (A1 and A2). Positive controls were performed using glyceraldehyde 3-phosphate dehydrogenase primers (GAPDH) (Fig. 1). Negative controls were performed in the absence of cDNA with the primers used in these studies (data not shown). Human umbilical artery smooth muscle expresses significant amounts of four families: PDE1, PDE3, PDE4 and PDE5, concretely the products of the PDE1B, PDE3A, PDE3B, PDE4C, PDE4D and PDE5A genes (Fig. 1). Concerning PDE5A, two products with different size of PDE5A were amplified indicating the existence of two splice variants (Fig. 1). Expression of products from PDE2 and PDE7 families was not detected.

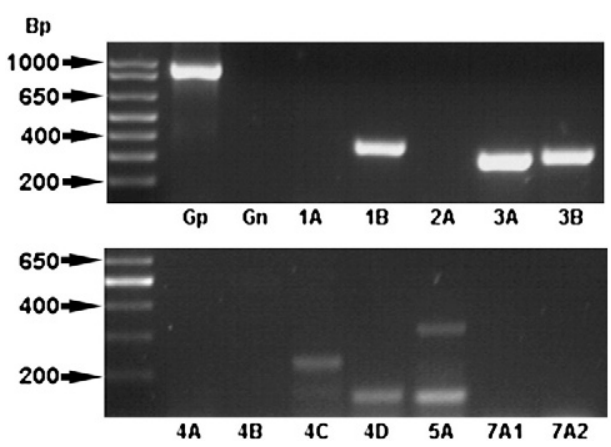

Fig. 1. Expression analysis of different phosphodiesterase (PDE) subtypes. Reverse transcriptase PCR analysis was performed using mRNA from human umbilical artery smooth muscle layers. The PCR products were analyzed on a $2.0 \%$ agarose gel and pictures of the ethidium bromide stained gels are shown. The PCR primers used for this analysis and expected length of the PCR products are described in Methods. Positive (Gp) and negative (Gn) controls were performed using glyceraldehyde 3-phosphate dehydrogenase primers. The expression of PDE1A (1A), PDE1B (1B), PDE2A (2A), PDE3A (3A), PDE3B (3B), PDE4A (4A), PDE4B (4B), PDE4C (4C), PDE4D (4D), PDE5A (5A), PDE7A1 (7A1) and PDE7A2 (7A2) was analysed. Positions of molecular weight markers are indicated in base pairs (Bp).

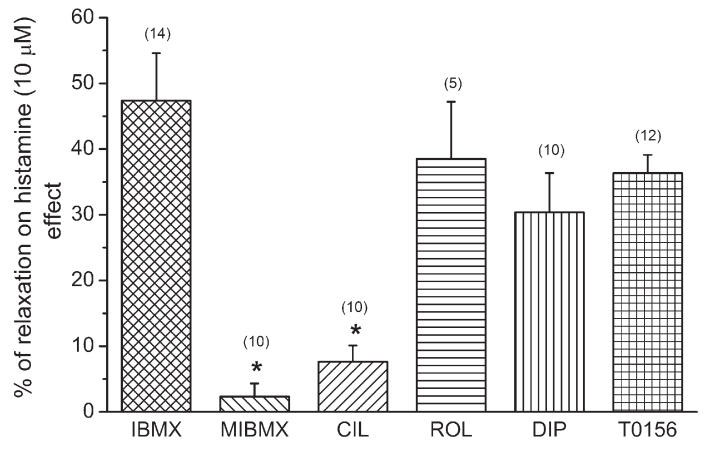

Fig. 2. Relaxation induced by PDE inhibitors on histamine-contracted arteries. The percent of inhibition induced by IBMX $(50 \mu \mathrm{M})$, MIBMX $(20 \mu \mathrm{M})$, cilostamide (CIL, $1 \mu \mathrm{M})$, rolipram (ROL, $1 \mu \mathrm{M})$, dipyridamole (DIP, $3 \mu \mathrm{M}$ ) and T0156 $(1 \mu \mathrm{M})$ on the histamine $(10 \mu \mathrm{M})$ effect is shown. The bars represent the means and the lines the S.E.M. of the numbers of experiments indicated near the bars. Statistical significant differences versus IBMX effect was performed using one-way ANOVA with Dunnet's post hoc test $(P<0.05)$.

\subsection{Effect of PDE inhibition and cyclases stimulation on histamine-contracted arteries}

Human umbilical artery rings without endothelium were contracted by histamine $(10 \mu \mathrm{M})$ and 5 -HT $(1 \mu \mathrm{M})$ and the effect of several PDE inhibitors on these contractions was analysed. Steady contractions were attained after 5-10 $\mathrm{min}$ in presence of 5-HT $(1 \mu \mathrm{M})$ or histamine $(10 \mu \mathrm{M})$ and the tension obtained was $1794.8 \pm 71.7$ and $1292.0 \pm 61.8 \mathrm{mg}$ respectively, which were significantly different $(P<0.05)$. Concerning the drugs stimulating the cyclases or inhibiting the PDEs, the time to reach the relaxing effects for each concentration was between 4 and $15 \mathrm{~min}$. The application of IBMX relaxed histaminecontracted arteries on $47.4 \pm 7.2 \%$ indicating the effect of the inhibition of all PDE types (Fig. 2). This effect seems to be mainly due to PDE4 and PDE5 inhibition because only rolipram

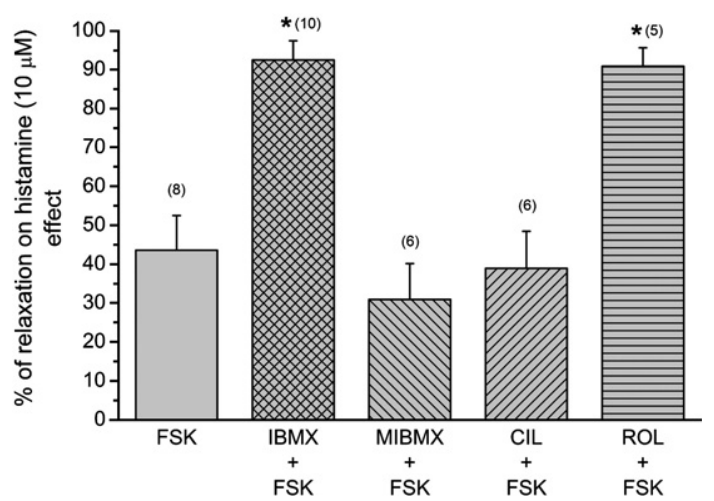

Fig. 3. Relaxation induced by adenylate cyclase stimulation in presence and absence of PDE inhibitors on histamine-contracted arteries. The percent relaxation induced by forskolin (FSK, $10 \mu \mathrm{M}$ ) alone or in presence of IBMX $(50 \mu \mathrm{M}), \operatorname{MIBMX}(20 \mu \mathrm{M})$, cilostamide $(\mathrm{CIL}, 1 \mu \mathrm{M})$ or rolipram (ROL, $1 \mu \mathrm{M})$ on histamine $(10 \mu \mathrm{M})$ effect is shown. The bars represent the means and the lines the S.E.M. of the numbers of experiments indicated near the bars. Statistical significant differences versus FSK effect was performed using one-way ANOVA with Dunnet's post hoc test $(P<0.05)$. 

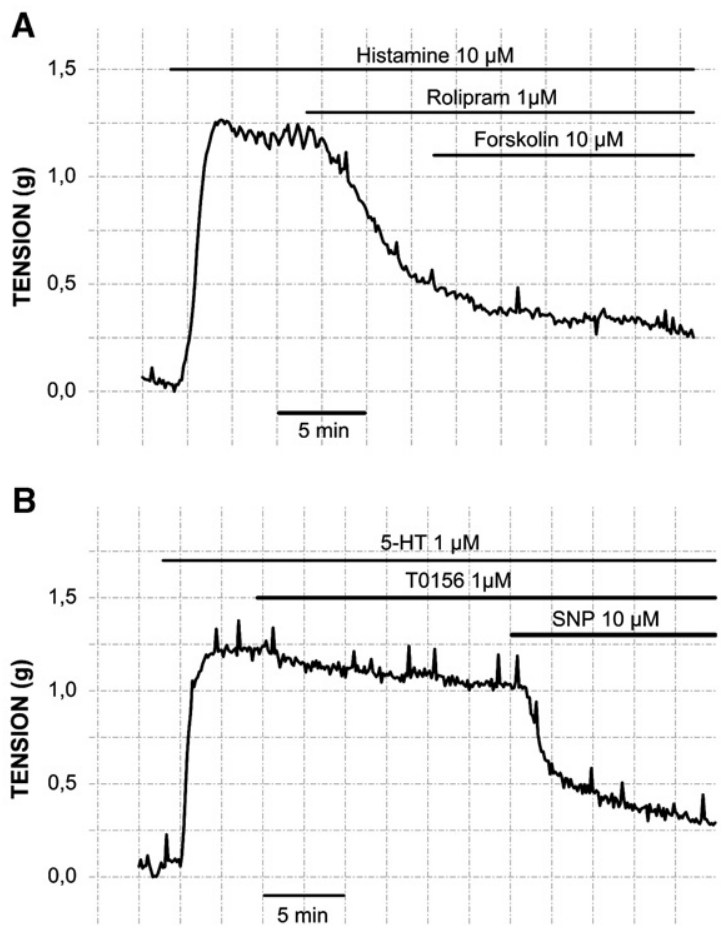

Fig. 4. Original record of two tension experiments with human umbilical artery rings. A) The artery rings were contracted by histamine $(10 \mu \mathrm{M})$ and relaxed by rolipram $(1 \mu \mathrm{M})$ alone and in presence of forskolin $(10 \mu \mathrm{M})$; B) The artery rings were contracted by 5 -HT $(1 \mu \mathrm{M})$ and relaxed by T0156 $(1 \mu \mathrm{M})$ alone and in presence of SNP $(10 \mu \mathrm{M})$.

(39.0 $\pm 8.7 \%)$, dipyridamole (30.4 $\pm 6.0 \%)$ and T0156 (36.3 \pm $2.8 \%$ ) induced effects comparable to that of IBMX (Fig. 2). The relaxation induced by MIBMX and cilostamide on histaminecontracted arteries was significantly lower than the observed with IBMX $(P<0.05)$. Ethanol, the vehicle used to dissolve the PDE inhibitors did not have any effect at the concentrations used (data not shown).

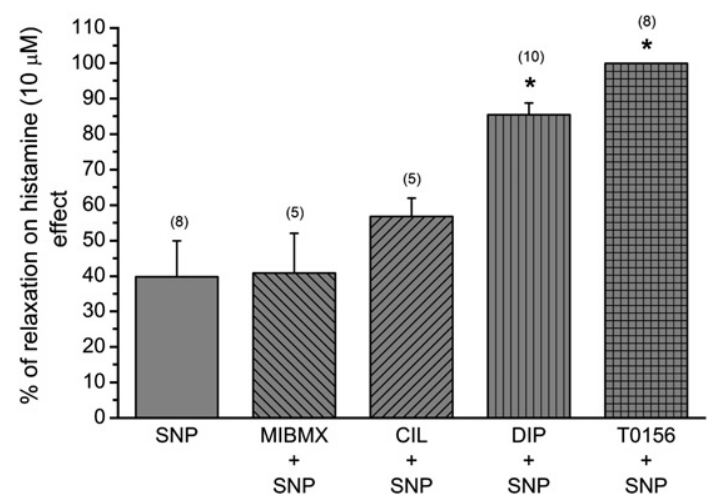

Fig. 5. Relaxation induced by guanylate cyclase stimulation in presence and absence of PDE inhibitors on histamine-contracted arteries. The percent relaxation induced by SNP $(10 \mu \mathrm{M})$ alone or in presence of MIBMX $(20 \mu \mathrm{M})$, cilostamide (CIL, $1 \mu \mathrm{M})$, dipyridamole (DIP, $3 \mu \mathrm{M})$ or T0156 $(1 \mu \mathrm{M})$ on histamine $(10 \mu \mathrm{M})$ effect is shown. The bars represent the means and the lines the S.E.M. of the numbers of experiments indicated near the bars. Statistical significant differences versus SNP effect was performed using one-way ANOVA with Dunnet's post hoc test $(P<0.05)$.

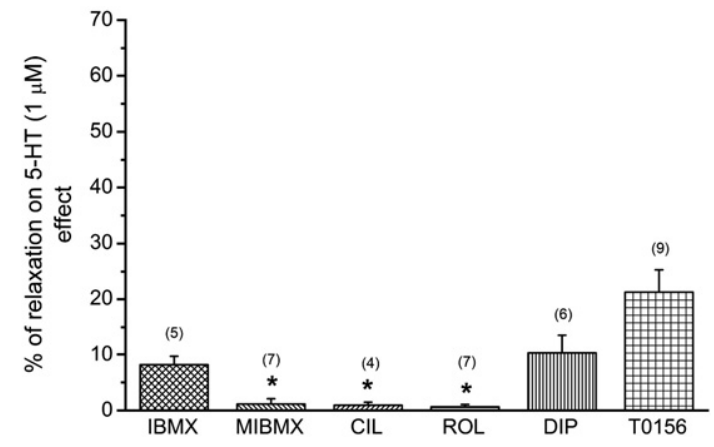

Fig. 6. Relaxation induced by PDE inhibitors on 5-HT contracted arteries. The percent of inhibition induced by IBMX $(50 \mu \mathrm{M})$, MIBMX $(20 \mu \mathrm{M})$, cilostamide (CIL, $1 \mu \mathrm{M}$ ), rolipram (ROL, $1 \mu \mathrm{M}$ ), dipyridamole (DIP, $3 \mu \mathrm{M}$ ) and T0156 $(1 \mu \mathrm{M})$ on the 5 -HT $(1 \mu \mathrm{M})$ effect is shown. The bars represent the means and the lines the S.E.M. of the numbers of experiments indicated near the bars. Statistical significant differences versus IBMX effect was performed using oneway ANOVA with Dunnet's post hoc test $(P<0.05)$.

When forskolin was used to increase cAMP levels, this adenylate cyclase activator relaxed $43.6 \pm 8.9 \%$ the histamineinduced contraction (Fig. 3). The inhibitors of PDEs degrading cAMP were further added to forskolin. Only IBMX and rolipram significantly increased the forskolin effect $(P<$ 0.05 ) and almost completely relaxed the human umbilical artery $(92.5 \pm 4.9 \%$ and $90.9 \pm 4.7 \%$, respectively). PDE1 and PDE3 inhibitors did not increase the forskolin effect on histamine contraction $(P>0.05)$ (Fig. 3). Fig. 4A shows a record of an experiment in which histamine-contracted arteries were relaxed by rolipram and this effect is increased when forskolin is further added.

When SNP was used to increase cGMP levels, this guanylate cyclase activator induced a relaxation of $39.8 \pm 10.1 \%$ on the histamine contraction. The inhibitors of PDEs degrading cGMP were further added and only the PDE5 selective inhibitors (dipyridamole and T0156) increase significantly $(P<0.05)$ the

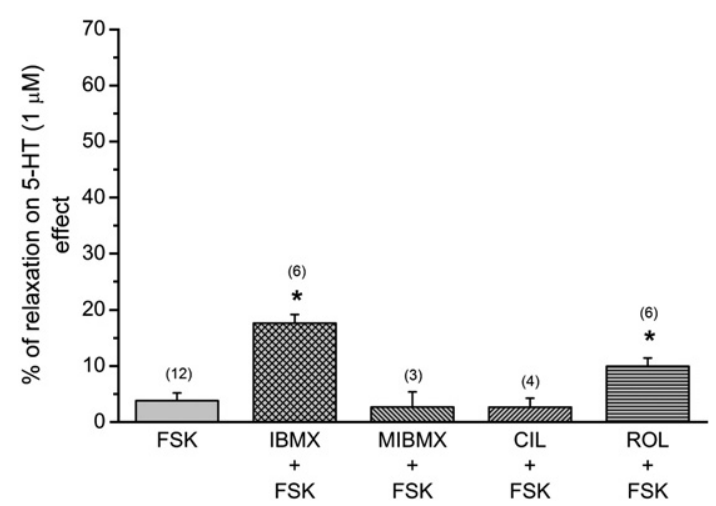

Fig. 7. Relaxation induced by adenylate cyclase stimulation in presence and absence of PDE inhibitors on 5-HT contracted arteries. The percent relaxation induced by forskolin (FSK, $10 \mu \mathrm{M})$ alone or in presence of IBMX $(50 \mu \mathrm{M})$, MIBMX $(20 \mu \mathrm{M})$, cilostamide (CIL, $1 \mu \mathrm{M})$ or rolipram (ROL, $1 \mu \mathrm{M})$ on 5-HT $(1 \mu \mathrm{M})$ effect is shown. The bars represent the means and the lines the S.E.M. of the numbers of experiments indicated near the bars. Statistical significant differences versus FSK effect was performed using one-way ANOVA with Dunnet's post hoc test $(P<0.05)$. 
SNP relaxant effect (Fig. 5). PDE1 and PDE3 inhibitors did not significantly increase the SNP effect on histamine-contracted arteries $(P>0.05)$.

\subsection{Effect of PDE inhibition and cyclases stimulation on 5-HT contracted arteries}

Our results demonstrated the existence of a lower relaxing effect of the PDE inhibitors on 5-HT contracted arteries, even in the presence of forskolin or SNP (Figs. 6, 7 and 8). The application of IBMX relaxed 5-HT contracted human umbilical artery on $8.2 \pm 1.5 \%$ (Fig. 6). Only PDE5 inhibition by dipyridamole or T0156 elicited similar effects than that of IBMX $(P>0.05)$. As showed in the Fig. 6, the effects induced by MIBMX, cilostamide and rolipram on 5-HT contracted arteries were weak and significantly lower than that observed with IBMX $(P<0.05)$. Ethanol, the vehicle used to dissolve the PDE inhibitors did not have any effect at the concentrations used (data not shown).

The activation of adenylate cyclase by forskolin relaxed 5HT contracted human umbilical artery in $3.9 \pm 1.4 \%$. When inhibitors of PDEs hydrolysing cAMP were added, the forskolin effect was only significantly increased by IBMX $(17.7 \pm 1.5 \%)$ and rolipram $(10.0 \pm 1.5 \%)(P<0.05)$ (Fig. 7). Like previously shown for histamine-contracted arteries, PDE1 and PDE3 inhibitors did not increase the forskolin effect $(P>0.05)$ on 5HT contracted arteries $(P>0.05)$.

The relaxing effect of SNP on 5-HT contracted human umbilical artery was $23.3 \pm 4.8 \%$ (Fig. 8 ). Concerning the PDEs specifically degrading cGMP, the SNP relaxing effect was only significantly augmented in the presence of the PDE5 inhibitor T0156 (52.0 $\pm 7.0 \%)(P<0.05)$. Fig. 4B shows a record of an experiment in which 5-HT contracted arteries are relaxed by T0156 and this effect is increased when SNP is further added.

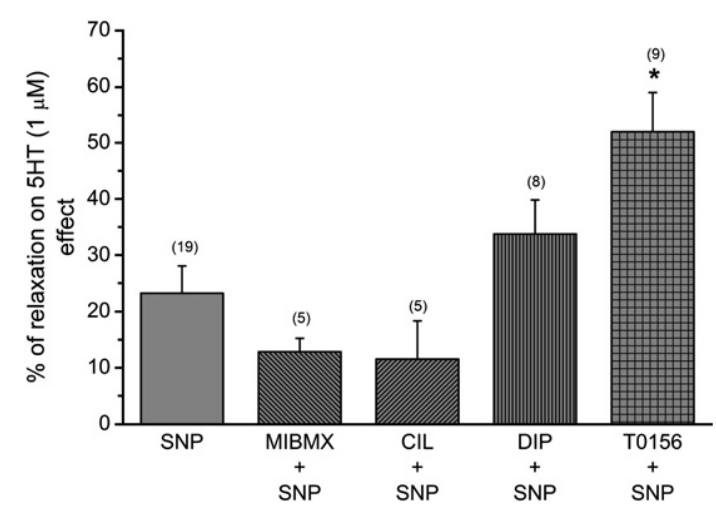

Fig. 8. Relaxation induced by guanylate cyclase stimulation in presence and absence of PDE inhibitors on 5-HT contracted arteries. The percent relaxation induced by SNP $(10 \mu \mathrm{M})$ alone or in presence of MIBMX $(20 \mu \mathrm{M})$, cilostamide (CIL, $1 \mu \mathrm{M})$, dipyridamole (DIP, $3 \mu \mathrm{M})$ or T0156 $(1 \mu \mathrm{M})$ on 5-HT $(1 \mu \mathrm{M})$ effect is shown. The bars represent the means and the lines the S.E.M. of the numbers of experiments indicated near the bars. Statistical significant differences versus SNP effect was performed using one-way ANOVA with Dunnet's post hoc test $(P<0.05)$.

\section{Discussion}

The present study investigates the involvement of different PDE families in the relaxation mediated by the cyclic nucleotides in human umbilical artery. The precise regulation of the intracellular levels of cAMP and cGMP plays an important role in many physiological processes, including vascular smooth muscle contractility (Matsumoto et al., 2003). As cyclic nucleotides can modulate the vascular smooth muscle tone, the PDEs have become an attractive target for drug development, due to the existence of different PDE isozymes which are differently regulated and distinctly expressed in the cardiovascular system. Concerning the fetoplacental circulation, cyclic nucleotide increase was appointed as inducing vasodilatation (Amit et al., 1998; Downing et al., 2004), however the PDE types modulating this effect were not described yet.

Several authors have reported that forskolin, a direct stimulator of adenylate cyclase, induced relaxation of different arteries such as canine colonic artery (Barnette et al., 1993), human dorsal artery (Segarra et al., 2002), human pulmonary artery (Rabe et al., 1994) and human placental vessels (Figueroa et al., 2000). These findings suggest that the elevation of cAMP plays a functional role in the regulation of vascular smooth muscle tone. Our results also showed that forskolin induces relaxation of contracted human umbilical arteries, and the effect of this adenylate cyclase stimulator on histamine-contracted arteries was bigger than on 5-HT contracted arteries. The different receptors implicated in the contractile effect of these agents could explain these results. The contractile effect of 5HT in denuded human umbilical artery seems to be due to the activation of two receptors: $5-\mathrm{HT}_{1 \mathrm{~B}}$ which activation inhibits adenylate cyclase; and 5- $\mathrm{HT}_{2 \mathrm{~A}}$ which activation increases inositol 1,4,5-triphosphate ( $\left.\mathrm{IP}_{3}\right)$ levels (Lovren et al., 1999). The contraction of human umbilical artery induced by histamine is due to the activation of $\mathrm{H}_{1}$ receptors, which induces increase of $\mathrm{IP}_{3}$ levels (Hawley et al., 1995). However, the expression of $\mathrm{H}_{2}$ receptors in human umbilical smooth muscle cells was also reported (Schneider et al., 2004) and the activation of these receptors stimulates adenylate cyclase. Thus, histamine and 5HT can increase $\mathrm{IP}_{3}$ levels, but histamine can also increase cAMP basal levels meanwhile 5-HT could have the opposite effect and this could be the cause of the lower relaxant effect of forskolin on 5-HT contracted arteries.

Sodium nitroprusside, a guanylate cyclase stimulator, induced relaxation of contracted human umbilical arteries, indicating that the elevation of intracellular cGMP plays a functional role in the regulation of vascular smooth muscle tone. The vasodilator effects of cGMP increase are bigger that the cAMP increase in 5HT contracted arteries (Figs. 7 and 8) indicating that the cGMP pathway is more efficient relaxing the 5-HT-induced contractions. The lower relaxing efficiency of cAMP on 5-HT contracted arteries could be due to the activation of $5-\mathrm{HT}_{1 \mathrm{~B}}$ receptors which activation inhibits adenylate cyclase. Other authors also observed the vasodilator properties of cGMP in canine colonic artery (Barnette et al., 1993), human pulmonary artery (Rabe et al., 1994), human placental vessels (Figueroa et al., 2000) and human umbilical artery (Lovren and Triggle, 2000). Furthermore, Amit 
et al. demonstrated that sublingual administration of isosorbide dinitrate, a nitrovasodilator, reduced maternal blood pressure and uterine arterial resistance (Amit et al., 1998). Our results also showed that SNP-induced relaxation on histamine-contracted arteries was bigger than on 5-HT contracted arteries. Lovren and Tiggle also observed that SNP induced relaxation was lower in human umbilical artery contracted with 5-HT when compared with arteries contracted with a thromboxane analogue (Lovren and Triggle, 2000).

The enzymes degrading cyclic nucleotides are the PDEs and the study of the isoforms that regulate cyclic nucleotide dependent vascular effects is crucial to analyze potential uses of the PDE inhibitors in therapeutics. Earlier studies using distinct vascular samples have provided direct or indirect evidence for the presence of products of six different PDE families in the vascular smooth muscle. The PDE1 (A and B) isoforms were detected in the smooth muscle isolated from the medial layer of human aorta (Rybalkin et al., 1997). PDE2 transcripts are not present in the human vascular smooth muscle and, at the vascular smooth muscle level, were only detected in porcine aorta smooth muscle (Matsumoto et al., 2003). The PDE3 expression was noticed in several human blood vessels, including placental smooth muscle (Omori and Kotera, 2007). PDE4 expression was observed in human pulmonary, mesenteric and femoral arteries (Maurice et al., 2003). PDE5 transcripts were detected in various human vascular tissues (Omori and Kotera, 2007). PDE7 was observed in human pulmonary artery and in endothelial cells (Matsumoto et al., 2003). Our results in human umbilical artery smooth muscle demonstrated the presence of transcripts encoding products from the following subtypes: PDE1B, PDE3A, PDE3B, PDE4C, PDE4D and PDE5A. It is interesting to note that two PCR products were obtained for PDE5A. Two alternate exons are present in the N-terminal region amplified giving the possibility of expressing different products with similar size like the ones obtained in our experiments: the smaller containing 140 base pairs and the bigger with 320 base pairs (Lin et al., 2006). Thus, the expression of products from four PDE families was detected in the human umbilical artery smooth muscle.

The use of a broad-spectrum PDE inhibitor (IBMX) resulted in a clear reduction in the histamine-induced contractility on human umbilical artery. The IBMX effect on 5-HT contracted arteries was almost five times lower than on histamine-contracted arteries. As already suggested before, these differences can be due to the different pathways implicated in the activation of histamine and 5HT receptors. To analyse the functionality of the PDE types in the regulation of the contractility of human umbilical artery, selective inhibitors of the PDE families expressed in this artery were used. Because at the micromolar concentrations the PDE5 inhibitors are not specific, two different inhibitors were used. T0156 inhibits PDE5 and PDE6 (Mochida et al., 2002b). Dipyridamol can inhibit PDE5, PDE6, PDE8 and PDE10 (Hetman et al., 2000) and can also inhibit the adenosine transport (Rubin et al., 2000). Rolipram, dipyridamole and T0156, but not MIBMX nor cilostamide, relaxed histamine-contracted human umbilical artery in a similar degree than IBMX. The relaxant effect of dipyridamole and T0156 on 5-HT-induced contraction was also similar than that obtained with IBMX. These results suggest a main role of PDE4 and PDE5 in the modulation of the cyclic nucleotide dependent relaxation.

The sequential addition of forskolin and inhibitors of the PDE families degrading cAMP allowed the study of the role of these isoforms on the cAMP-dependent relaxation of human umbilical artery. The addition of IBMX or rolipram to forskolin increased the effect of the adenylate cyclase stimulator and almost completely relaxed the human umbilical artery contracted by histamine. This suggests that PDE4 is the key PDE regulating the cAMP-dependent relaxation. Even if the relaxant effects are lesser, the results obtained with 5-HT contracted arteries confirmed the importance of PDE4 in the regulation of human umbilical artery smooth muscle contractility, because only rolipram and IBMX significantly increased the forskolin vasodilator effect. Some authors suggested that PDE4 is the predominant family implicated in the cAMP-dependent regulation of canine basilar artery contractility (Willette et al., 1997). Other authors, using human cavernous arteries contracted with norepinephrine, observed that cAMP-dependent relaxation is regulated by PDE4 and PDE3 (Waldkirch et al., 2005).

The sequential addition of SNP, a NO donor, and inhibitors of the PDE families degrading cGMP allowed the study of the role of these isoforms on the cGMP-dependent relaxation of human umbilical artery. Further addition of selective PDE5 inhibitors (dipyridamole or T0156) to SNP revealed a significant increase in the relaxation of histamine-contracted arteries, an effect that was not observed with PDE1 or PDE3 inhibitors. These data suggest that PDE5 is involved in the regulation of the relaxation associated to cGMP. Is well known that NO stimulates cGMP formation by activation of soluble guanylate cyclase, which in turn has vasodilatatory actions (Kristek et al., 2007). In this context, the NO/cGMP pathway was involved in human umbilical artery vasodilatation (Lovren and Triggle, 2000). The data obtained with 5-HT contracted rings also confirmed the major role of PDE5 modulating cGMP-dependent vasodilatation, because dipyridamole and T0156 were the unique PDE inhibitors that increased the SNP relaxing effect. These data also shows that the vasodilator effects of the combination of SNP and T0156 are bigger than that induced by the mixture of forskolin and rolipram (Figs. 7 and 8), confirming that the 5-HT-induced contractions are more efficiently relaxed by the cGMP pathway. The involvement of PDE5 in the modulation of cGMP-dependent relaxation was also observed by Rabe et al. (1994) in human pulmonary artery contracted with prostaglandin $\mathrm{F}_{2 \alpha}$, although this authors also proposed a role of the PDE3 in this action (Rabe et al., 1994). Waldkirch et al. (2005), working with human cavernous arteries contracted by norepinephrine, implicated PDE5 in the regulation of the vasodilatation associated to cGMP (Waldkirch et al., 2005). Some authors proposed the use of nitrovasodilators or PDE5 inhibitors for pregnant women with vascular complications (Amit et al., 1998; Downing et al., 2004; Pinheiro da Costa et al., 2006).

In summary, our results demonstrate that the increase of cAMP and/or cGMP levels in the human umbilical vascular smooth muscle induces relaxation. Four families of PDE are 
expressed in this smooth muscle: PDE1, PDE3, PDE4 and PDE5. Between these families, PDE4 and PDE5 are the key enzymes involved in the regulation of human umbilical artery relaxation associated to cAMP and cGMP, respectively. PDE1 and PDE3 activities seem to be not relevant to this regulation. Our results suggest that, due to the functional importance of these PDE isozymes, the selective PDE4 and PDE5 inhibitors may represent a therapeutic tool for the treatment of some pathologies as pre-eclampsia and hypertension in pregnancy.

\section{Acknowledgements}

We thank the donor mothers and the Gynaecology-Obstetrics Department staff of "Centro Hospitalar da Cova da Beira" (Covilhã, Portugal) for their disinterested collaboration and the FCT (Fundação para a Ciência e a Tecnologia) for supporting the fellowship grant SFRH/BDE/15532/2004.

\section{References}

Amit, A., Thaler, I., Paz, Y., Itskovitz-Eldor, J., 1998. The effect of a nitric oxide donor on Doppler flow velocity waveforms in the uterine artery during the first trimester of pregnancy. Ultrasound Obstet. Gynecol. 11, 94-98.

Barnette, M.S., Manning, C.D., Price, W.J., Barone, F.C., 1993. Initial biochemical and functional characterization of cyclic nucleotide phosphodiesterase isozymes in canine colonic smooth muscle. J. Pharmacol. Exp. Ther. 264, 801-812.

Beavo, J.A., 1995. Cyclic nucleotide phosphodiesterases: functional implications of multiple isoforms. Physiol. Rev. 75, 725-748.

Downing, J.W., Ramasubramanian, R., Johnson, R.F., Minzter, B.H., Paschall, R.L., Sundell, H.W., Engelhardt, B., Lewis, R., 2004. Hypothesis: selective phosphodiesterase-5 inhibition improves outcome in preeclampsia. Med. Hypotheses 63, 1057-1064.

Figueroa, R., Martinez, E., Fayngersh, R.P., Tejani, N., Mohazzab, H.K., Wolin, M.S., 2000. Alterations in relaxation to lactate and $\mathrm{H}_{2} \mathrm{O}_{2}$ in human placental vessels from gestational diabetic pregnancies. Am. J. Physiol. Heart Circ. Physiol. 278, H706-H713.

Gupta, R., Kumar, G., Kumar, R.S., 2005. An update on cyclic nucleotide phosphodiesterase (PDE) inhibitors: phosphodiesterases and drug selectivity. Methods Find. Exp. Clin. Pharmacol. 27, 101-118.

Hawley, J., Rubin, P.C., Hill, S.J., 1995. Distribution of receptors mediating phosphoinositide hydrolysis in cultured human umbilical artery smooth muscle and endothelial cells. Biochem. Pharmacol. 49, 1005-1011.

Hetman, J.M., Soderling, S.H., Glavas, N.A., Beavo, J.A., 2000. Cloning and characterization of PDE7B, a cAMP-specific phosphodiesterase. Proc. Natl. Acad. Sci. U. S. A. 97, 472-476.

Kristek, F., Koprdova, R., Cebova, M., 2007. Long-term effects of early administered sildenafil and NO donor on the cardiovascular system of SHR J. Physiol. Pharmacol. 58, 33-43.

Leung, S.W., Quan, A., Lao, T.T., Man, R.Y., 2006. Efficacy of different vasodilators on human umbilical arterial smooth muscle under normal and reduced oxygen conditions. Early Hum. Dev. 82, 457-462.

Lillestll, I.K., Helle, K.B., Aardal, S., 1998. Relaxing effects of cyclic GMP and cyclic AMP-enhancing agents on the long-lasting contraction to endothelin1 in the porcine coronary artery. Scand. J. Clin. Lab. Invest. 58, 625-634.

Lin, C.S., Lin, G., Xin, Z.C., Lue, T.F., 2006. Expression, distribution and regulation of phosphodiesterase 5. Curr. Pharm. Des. 12, 3439-3457.

Lovren, F., Li, X.F., Lytton, J., Triggle, C., 1999. Functional characterization and m-RNA expression of 5-HT receptors mediating contraction in human umbilical artery. Br. J. Pharmacol. 127, 1247-1255.
Lovren, F., Triggle, C., 2000. Nitric oxide and sodium nitroprusside-induced relaxation of the human umbilical artery. Br. J. Pharmacol. 131, 521-529.

Lugnier, C., 2006. Cyclic nucleotide phosphodiesterase (PDE) superfamily: a new target for the development of specific therapeutic agents. Pharmacol. Ther. 109, 366-398.

Matsumoto, T., Kobayashi, T., Kamata, K., 2003. Phosphodiesterases in the vascular system. J. Smooth Muscle Res. 39, 67-86.

Maurice, D.H., Palmer, D., Tilley, D.G., Dunkerley, H.A., Netherton, S.J., Raymond, D.R., Elbatarny, H.S., Jimmo, S.L., 2003. Cyclic nucleotide phosphodiesterase activity, expression, and targeting in cells of the cardiovascular system. Mol. Pharmacol. 64, 533-546.

Mochida, H., Inoue, H., Takagi, M., Noto, T., Yano, K., Kikkawa, K., 2002a. Sildenafil and T-1032, phosphodiesterase type 5 inhibitors, showed a different vasorelaxant property in the isolated rat aorta. Eur. J. Pharmacol. 440, $45-52$.

Mochida, H., Takagi, M., Inoue, H., Noto, T., Yano, K., Fujishige, K., Sasaki, T., Yuasa, K., Kotera, J., Omori, K., Kikkawa, K., 2002b. Enzymological and pharmacological profile of T-0156, a potent and selective phosphodiesterase type 5 inhibitor. Eur. J. Pharmacol. 456, 91-98.

Noguera, M.A., Ivorra, M.D., Lugnier, C., D’Ocon, P., 2001. Role of cyclic nucleotide phosphodiesterase isoenzymes in contractile responses of denuded rat aorta related to various $\mathrm{Ca} 2+$ sources. Naunyn Schmiedebergs Arch. Pharmacol. 363, 612-619.

Omori, K., Kotera, J., 2007. Overview of PDEs and their regulation. Circ. Res. 100, 309-327.

Pinheiro da Costa, B.E., Scocco, C., Poli de Figueiredo, C.E., Guimaraes, J.A., 2006. Increased serum phosphodiesterase activity in women with preeclampsia. BJOG 113, 577-579.

Rabe, K.F., Tenor, H., Dent, G., Schudt, C., Nakashima, M., Magnussen, H., 1994. Identification of PDE isozymes in human pulmonary artery and effect of selective PDE inhibitors. Am. J. Physiol. 266, L536-L543.

Rubin, L.J., Johnson, L.R., Dodam, J.R., Dhalla, A.K., Magliola, L., Laughlin, M.H., Jones, A.W., 2000. Selective transport of adenosine into porcine coronary smooth muscle. Am. J. Physiol. Heart Circ. Physiol. 279, H1397-H1410.

Rybalkin, S.D., Bornfeldt, K.E., Sonnenburg, W.K., Rybalkina, I.G., Kwak, K.S., Hanson, K., Krebs, E.G., Beavo, J.A., 1997. Calmodulin-stimulated cyclic nucleotide phosphodiesterase (PDE1C) is induced in human arterial smooth muscle cells of the synthetic, proliferative phenotype. J. Clin. Invest. 100, 2611-2621

Rybalkin, S.D., Yan, C., Bornfeldt, K.E., Beavo, J.A., 2003. Cyclic GMP phosphodiesterases and regulation of smooth muscle function. Circ. Res. 93, 280-291.

Schneider, A., Riess, P., Elbers, A., Neugebauer, E., Schaefer, U., 2004. Polyclonal anti-histamine $\mathrm{H} 2$ receptor antibodies detect differential expression of $\mathrm{H} 2$ receptor protein in primary vascular cell types. Inflamm. Res. 53, 223-229.

Segarra, G., Medina, P., Vila, J.M., Martinez-Leon, J.B., Domenech, C., Prieto, F., Lluch, S., 2002. Relaxation induced by milrinone and rolipram in human penile arteries and veins. Eur. J. Pharmacol. 444, 103-106.

Tilley, D.G., Maurice, D.H., 2002. Vascular smooth muscle cell phosphodiesterase (PDE) 3 and PDE4 activities and levels are regulated by cyclic AMP in vivo. Mol. Pharmacol. 62, 497-506.

Waldkirch, E., Uckert, S., Yildirim, H., Sohn, M., Jonas, U., Stief, C.G., Andersson, K.E., Hedlund, P., 2005. Cyclic AMP-specific and cyclic GMPspecific phosphodiesterase isoenzymes in human cavernous arteriesimmunohistochemical distribution and functional significance. World $\mathrm{J}$. Urol. 23, 405-410.

Willette, R.N., Shiloh, A.O., Sauermelch, C.F., Sulpizio, A., Michell, M.P., Cieslinski, L.B., Torphy, T.J., Ohlstein, E.H., 1997. Identification, characterization, and functional role of phosphodiesterase type IV in cerebral vessels: effects of selective phosphodiesterase inhibitors. J. Cereb. Blood Flow Metab. 17, 210-219. 